\title{
Both Cyclophilin Inhibitors and Direct-Acting Antivirals Prevent PKR Activation in HCV-Infected Cells
}

\author{
Michael Bobardt, Udayan Chatterji, Precious Lim, Katarzyna Gawlik and Philippe Gallay*
}

Department of Immunology \& Microbial Science, The Scripps Research Institute, La Jolla, California 92037, USA

\begin{abstract}
We and others demonstrated that the contact between NS5A and the host factor CypA is critical for HCV replication. CypI, by disrupting NS5A-CypA complexes, block HCV replication both in vitro and in patients. Since NS5A also binds to PKR, a central component of the IFN response, we investigated the possibility of a relationship between CypA, NS5A and PKR in the IFN response to HCV. HCV-infected cells treated with CypI, DAAs or IFN were analyzed for the expression and activation of various components of the innate response. We found that CypI (cyclosporine A, alisporivir, NIM811 and sanglifehrins), drastically prevented the activation/phosphorylation, but not the expression of IFN-induced PKR in HCV-infected cells. CypI had no effect on the expression or phosphorylation of other components of the innate response such as eiF2, NF-kB, IRF3, IRF9, STAT1 and STAT2, suggesting a specific effect on PKR. No significant activation of IFN-induced PKR was observed in the absence of HCV. Importantly, we found that several classes of DAAs such as NS3/4A protease, NS5B polymerase and NS5A inhibitors also prevented PKR activation. Furthermore, we found that PKR activation by the dsRNA mimic poly I:C cannot be prevented by CypI or DAAs. Our findings suggest that CypI do not have a unique effect on PKR activation, but rather the suppression of HCV replication by any anti-HCV inhibitor, abrogates PKR activation induced by IFN. Moreover, they suggest that the accumulation of dsRNA intermediates allows HCV to exploit the activation of PKR to counteract the IFN response.
\end{abstract}

Keywords: CypI, DAAs, HCV, IFN response, NS5A, PKR.

\section{INTRODUCTION}

Chronic hepatitis C affects approximately 200 million people worldwide and is a leading cause of acute and chronic liver diseases [1] and 4 million new HCV infections occur each year [2, 3]. HCV accounts for two third of liver cancer and transplant cases in the developed world [4], and $12,000-15,000$ individuals are projected to die from $\mathrm{HCV}$ every year in the U.S. [5]. Until 2011, the combination of pegylated IFN $\alpha$ and RBV had a success rate of $\sim 80 \%$ in patients with GT2 and GT3, but only $~ 50 \%$ in patients with GT1 and most importantly causes severe side effects [6-10]. There is thus an imperative demand for the identification and development of supplementary anti-HCV agents with diversified mechanisms of action in order to deliver interchangeable therapies for patients who are poorly responsive or non-responsive to the pegylated IFN $\alpha / R B V$ standard of care [1,2]. A novel class of anti-HCV agents has recently emerged: the HTAs cyclophilin inhibitors [11-19]. Three cyclophilin inhibitors - alisporivir, NIM811 and SCY635 have been tested in early phase I and II studies [20-30]. Alisporivir is the most advanced in this class, with the largest number of patients exposed and currently is in clinical development as IFN-free treatment.

$\mathrm{We}$ and others obtained evidence that $\mathrm{HCV}$ relies on host CypA to replicate in human hepatoma cells [31-33] as well

*Address correspondence to this author at the Department of Immunology \& Microbial Science, IMM-9, The Scripps Research Institute, $10550 \mathrm{~N}$. Torrey Pines Rd., La Jolla, CA 92037, USA; Tel: (858) 784-8180;

Fax: (858) 784-8831; E-mail: gallay@ scripps.edu as in humanized mice [34]. Since CypA represents the major intracellular target for CypI such as cyclosporine A [35], it has been proposed that they block HCV replication by neutralizing the peptidyl-prolyl isomerase activity of CypA. We and others also demonstrated that CypA can interact directly with the HCV NS5A protein and that most importantly, CypI prevent NS5A-CypA interactions in a dose-dependent manner [36-39]. We also showed that the interaction between NS5A and CypA is conserved among $\mathrm{HCV}$ genotypes and that CypI block all these interactions [38]. These in vitro data are in accordance with the fact that CypI are pangenotypic both in vitro and in patients. There is thus a direct correlation between disrupting NS5A-CypA complexes and blocking HCV replication. The Lippens and the Hanoulle labs elegantly showed that CypA induces cistrans isomerization of several proline residues within the domains II and III of NS5A [36, 39, 40]. Interestingly, CypA and the NS5B polymerase share a common binding site on NS5A [41]. However, it remains obscure how CypA, by binding to NS5A and/or by isomerizing NS5A, potentiates $\mathrm{HCV}$ replication.

The IFN-inducible PKR plays multiple roles in a cell, in response to different stress situations. As a member of the ISGs, PKR was recognized as a factor in the antiviral action of IFN [42], due to its ability to control translation, through phosphorylation, of the $\alpha$ subunit of eIF2a. As such, PKR participates in the generation of stress granules or autophagy, and a number of viruses have developed strategies to inhibit its action. Mutations within the PKR-binding region of NS5A, including those within the ISDR, disrupt NS5A-PKR interactions [43]. Gale et al. showed that NS5A interacts in 
vitro with PKR [43]. Previous studies nicely demonstrated that NS5A is an RNA binding protein [44, 45], which can regulate the binding of PKR to the IRES of the HCV RNA [46]. Based on these findings, it has been proposed that the NS5A-PKR interaction serves as a target for therapeutic strategies against HCV. Since we and others obtained several lines of evidence suggesting that the NS5A-CypA interaction also represents an attractive target for the development of anti-HCV agents such as CypI, we asked in this study whether CypA and PKR act in concert to regulate $\mathrm{HCV}$ replication.

\section{MATERIAL AND METHODS}

\section{Compounds}

The HCV NS5A inhibitor daclatasvir (Bristol Myers Squibb), the HCV NS5B polymerase inhibitor sofosbuvir (Gilead), the HCV NS3 protease inhibitors boceprevir (Merck) and telaprevir (Vertex) and the HIV-1 reverse transcriptase inhibitor emtricitabine (Gilead) were all obtained from MedChemexpress (Princeton, NJ 08540, USA). Alisporivir and NIM811 were generously provided by Novartis, whereas cyclosporine A, sanglifehrins A and B were generously provided by Drs. Wilkinson and Gregory. Poly I:C was obtained from InvivoGen (San Diego, CA, USA).

\section{Replicons}

The GT2a subgenomic JFH-1 replicon was generously provided by Drs. T. Wakita and F. Chisari. The GT2a genomic luciferase reporter replicon Luc-Neo-JFH-1 was created as follows. The plasmid pFK-Luc-JFH1 was generously obtained from Drs. T. Wakita and T. Pietschmann [47, 48] and the XbaI site in the firefly luciferase gene, and the NotI site in the EMCV IRES were utilized to clone the Luciferase/Ubiquitin-NPT II fusion cassette out of pFK389I LucNeo (wild-type replicon from GT1b) (generous gift from Dr. R. Bartenschlager) [48, 49] and placed into the pFK-LucJFH1 plasmid, creating the full-length Luc-Neo-JFH-1 construct. Replicons were stably expressed in Huh7.5.1 cells under G418 selection.

\section{Antibodies}

Anti-PKR, anti-eiF2, anti-IRF3, anti-IRF9, anti-NF-kB and anti-OAS1 antibodies were obtained from Santa Cruz; the anti-phospho-PKR antibody was obtained from Abcam; anti-phospho-eiF2, anti-STAT1, anti-phospho-STAT1 antibody were obtained from Cell Signaling Technologies; the anti-NS5A antibody (9E10) was generously obtained by Dr. C. Rice; and anti-calnexin antibody was obtained from Sigma.

\section{PKR Activation}

Parental, genomic or subgenomic JFH-1-expressing Huh7.5.1 cells plated for $24 \mathrm{~h}$ were treated with or without CypI or direct-acting antivirals (daclatasvir, sofosbuvir, boceprevir, telaprevir and emtricitabine). Cells were then treated for $24 \mathrm{~h}$ with IFN $\alpha(300 \mathrm{U} / \mathrm{mL})$ and lysed. Lysates were standardized for protein content and analyzed by Western blotting for their content in various host and viral proteins.

\section{RESULTS}

\section{Alisporivir Prevents PKR Activation}

We chose the potent non-immunosuppressive CypI alisporivir to determine the effect of CypA neutralization on PKR activation. We also chose to employ the JFH-1 cell culture system for productive HCV infection [47, 50, 51] since it allows more direct measurements of the effects of the IFN response during the life cycle of the virus. To induce PKR activation, IFN $\alpha$ or IFN $\beta$ were added to HCV-infected cells pre-incubated with or without alisporivir. Twenty-four and $48 \mathrm{~h}$ post-IFN treatment, cells were washed and lysed. Lysates were analyzed for their content in various viral and host proteins. In accordance with the fact that $\mathrm{HCV}$ is sensitive to IFN in vitro, NS5A levels in JFH-1-infected cells treated with IFN $\alpha$ or IFN $\beta$ were significantly decreased 24 and $48 \mathrm{~h}$ post-IFN treatment (Fig. 1A). NS5A levels were profoundly reduced in alisporivir-treated cells compared to untreated cells (Fig. 1A), further suggesting that the CypI alisporivir, by interfering with viral RNA replication, suppresses the expression and production of $\mathrm{HCV}$ proteins such as NS5A. We obtained similar results for the HCV core (data not shown). We then looked at the expression of the non-phosphorylated form of PKR, also called the inactive form of PKR. A significant level of inactive/nonphosphorylated PKR pre-existed in JFH-1-infected cells and both IFN $\alpha$ and IFN $\beta$ increased its expression, whereas alisporivir alone had no effect (Fig. 1A). This confirms that $\mathrm{PKR}$ is an IFN-inducible protein and suggests that CypA neutralization does not regulate the expression of the inactive/non-phosphorylated form of PKR in an established infection. Importantly, we found that IFN $\alpha$ and IFN $\beta$ greatly promoted the levels of phosphorylated PKR, the active form of PKR 24 and $48 \mathrm{~h}$ post-IFN treatment (Fig. 1A). Remarkably, alisporivir prevented the IFN-mediated activation/phosphorylation of PKR (Fig. 1A). The IFN induction as well as the alisporivir-mediated inhibition of PKR activation appears to be specific for components of the innate response such as NF- $\kappa \mathrm{B}$, eIF2, its phosphorylated form (phospho-eiF2), IRF3 and IRF9 as well as STAT2 were not altered by alisporivir (Fig. 1A). Altogether these results demonstrate that CypA inhibition by alisporivir markedly decreases the IFN-induced activation/phosphorylation of PKR. These data provide the first link between HCV, CypA and PKR.

We then asked whether similar results can be observed using a subgenomic JFH-1 replicon. Similarly to the infectious genomic HCV replicon, we found that IFN upregulates the expression of PKR in cells replicating the subgenomic replicon (Fig. 1B). As for the genomic HCV replicon, we found that alisporivir prevents the IFN-induced activation/phosphorylation of PKR, has no effect of the expression of inactive/non-phosphorylated PKR and decreases the expression of HCV proteins such as NS5A (Fig. 1B). We obtained similar results for subgenomic replicons from other genotypes (1b, 3a and 4a) (data not shown). These data indicate that HCV structural proteins are not necessary for the activation of IFN-induced PKR by IFN.

We also examined whether the alisporivir impact on the inhibition of PKR activation is IFN concentration-dependent. 
A

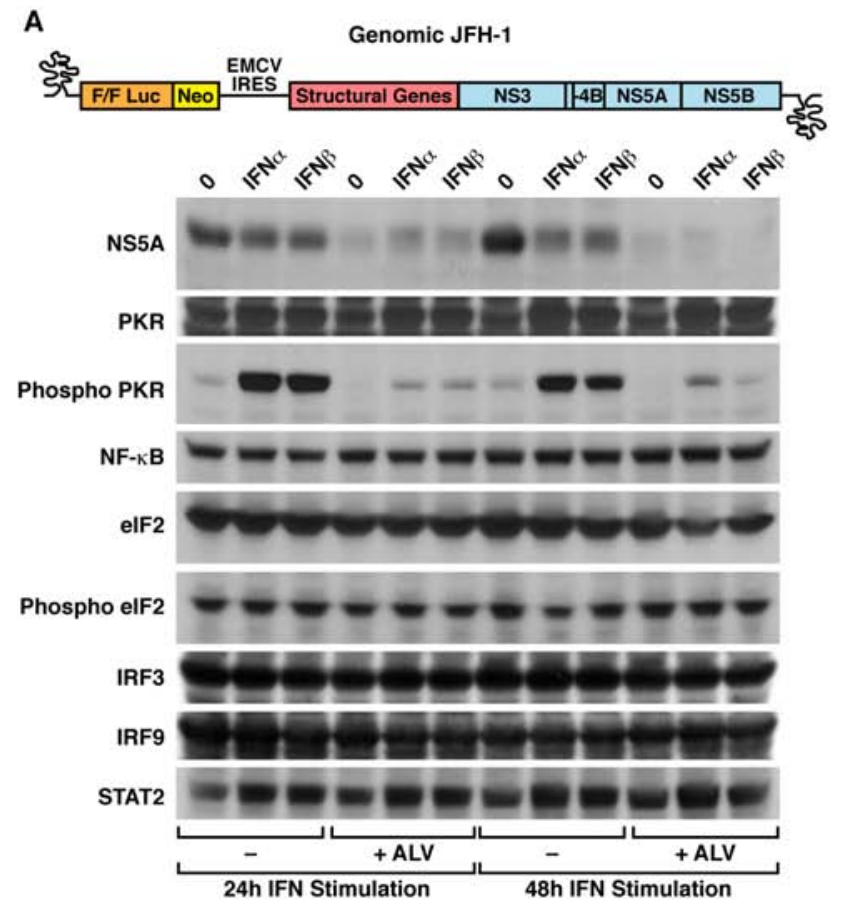

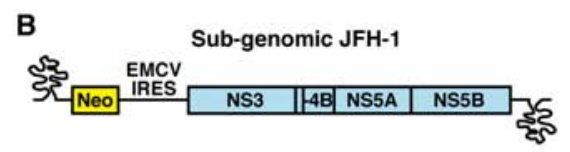

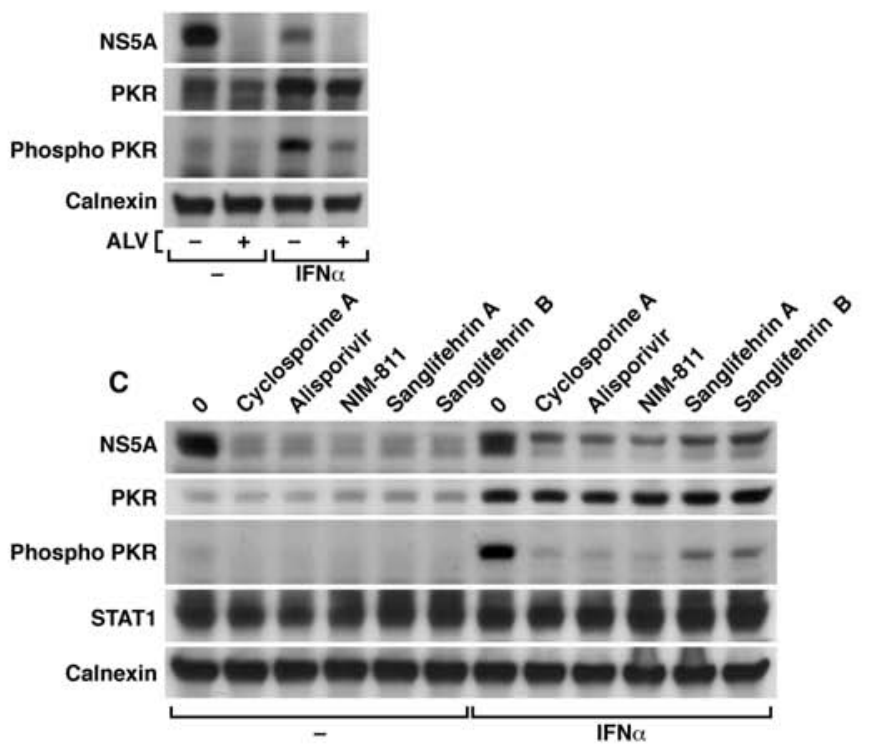

Fig. (1). CypI prevent IFN-induced PKR activation in HCV-infected cells. (A) Schematic diagram of genomic JFH-1 replicon used is depicted. JFH-1-infected Huh7.5.1 cells were treated with or without the CypI alisporivir (ALV) or NIM811, and subsequently treated with IFN $\alpha$ or $\beta$ for 24 or $48 \mathrm{~h}$. Cells were then lysed and analyzed for their content in viral and host proteins by Western blotting. (B) Schematic diagram of subgenomic JFH-1 replicon used is depicted. Same as (A) except that JFH-1 subgenomic replicon cells were used and treated with IFN $\alpha$ for $24 \mathrm{~h}$. (C) Same as (A) except that JFH-1-infected Huh7.5.1 cells were treated with a panel of CypI as well as with or without IFN $\alpha$ for $24 \mathrm{~h}$. Results are representative of 3-5 independent experiments.

$\mathrm{HCV}$-infected cells were treated with increasing concentrateions of IFN $\alpha$ (from 100 to $2700 \mathrm{U} / \mathrm{mL}$ ). We found that a low concentration of IFN $\alpha(100 \mathrm{U} / \mathrm{mL})$ sufficed to increase the expression of inactive/non-phosphorylated PKR, whereas an intermediate concentration of IFN $\alpha(300 \mathrm{U} / \mathrm{mL})$ gave the most profound activation of PKR (data not shown). Alisporivir reduced the IFN-mediated activation of PKR independently of the IFN dose (data not shown). We obtained similar results for IFN $\beta$ (type I), IFN $\gamma$ (type II) and IFN $\lambda$ (type III) (data not shown), suggesting that alisporivir prevents the activation of PKR independently of the type of IFN used for induction.

\section{Any CypI Prevents the Activation of IFN-Induced PKR}

We showed above that the treatment of HCV-infected cells with the CypI alisporivir suppresses PKR activation/ phosphorylation. We then asked whether this inhibition is specific to alisporivir or is mediated by any CypI. There are two main classes of CypI - i) cyclosporine A and derivates and ii) sanglifehrins and derivates - that both target the isomerase pocket of CypA. We chose cyclosporine A, the two non-immunosuppressive cyclosporine A analogs - alisporivir and NIM-811 - and sangliferins A and B. We employed the same experimental design as above except that we used IFN $\alpha$ exclusively and lysed HCV-infected cells $24 \mathrm{~h}$ postIFN treatment. All CypI significantly decreased NS5A levels, but did not affect the IFN-induced expression of inactive/non-phosphorylated PKR (Fig. 1C). However, each CypI efficiently suppressed PKR activation/phospho-rylation
(Fig. 1C). These data suggest that inhibition of CypA prohibits the activation of IFN-induced PKR in HCVinfected cells.

\section{DAAs Also Block PKR Activation}

We then asked whether anti-HCV agents other than CypI such as DAAs also modulate the activation of PKR. Specifically, we tested the effect of the protease inhibitors boceprevir and telaprevir as well as the NS5A inhibitor daclatasvir. We used as positive control the CypI alisporivir and as negative control the $\mathrm{HIV}-1$ reverse transcriptase inhibitor emtricitabine. All anti-HCV agents were potent. Indeed alisporivir, boceprevir, telaprevir and daclatasvir, but not the HIV-1 agent emtricitabine, suppressed the expression of $\mathrm{HCV}$ proteins such as NS5A (Fig. 2). Examination of calnexin levels showed that similar amounts of cell lysates were analyzed (Fig. 2). IFN elevates the expression of inactive/non-phosphorylated PKR and all inhibitors have no effect on it (Fig. 2). Similarly, IFN enhances the phosphorylation of STAT1 that is not influenced by the inhibitors (Fig. 2). Most importantly, we found that all antiHCV agents - alisporivir, boceprevir, telaprevir and daclatasvir - but not the HIV-1 inhibitor emtricitabine, prevent PKR activation/phosphorylation (Fig. 2). This finding is critical because it demonstrates that the inhibition of PKR activation is not unique to CypI, but is shared by any anti-HCV agents.

The latter finding led us to postulate that there is a direct correlation between PKR activation and viral replication. To test this hypothesis, we conducted similar experiments in 


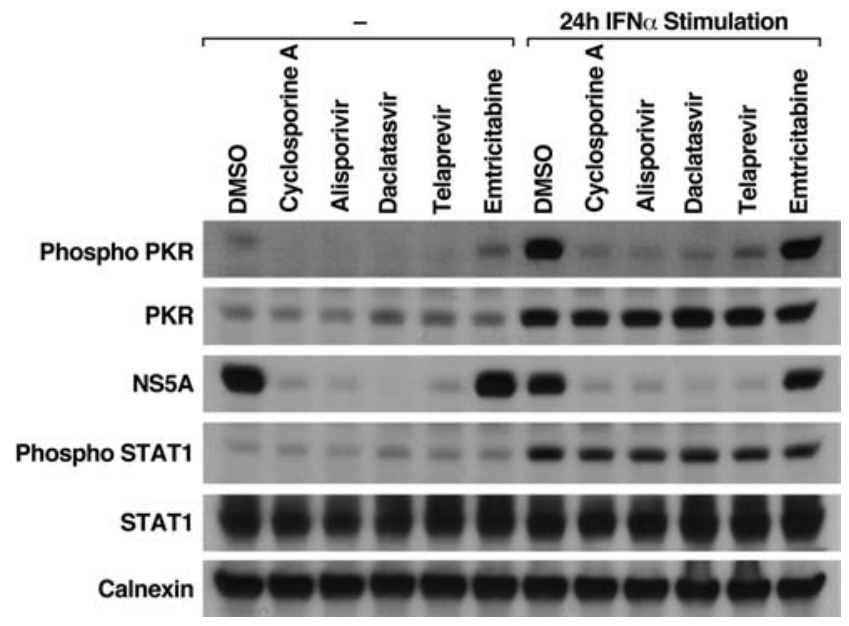

Fig. (2). The PKR activation block is not unique to CypI, DAAs also prevent the IFN-induced PKR activation in $\mathrm{HCV}$-infected cells. Same as (Fig. 1c) except that JFH-1-infected Huh7.5.1 cells were treated with or without CypI (cyclosporine A and alisporivir), DAAs (the HCV NS5A inhibitor daclatasvir and the HCV protease inhibitor telaprevir) and an HIV-1 inhibitor (reverse transcriptase inhibitor emtricitabine). Results are representative of 4 independent experiments.

both non-infected and HCV-infected cells. We chose as anti$\mathrm{HCV}$ agents - alisporivir, the NS5A inhibitor daclastavir and the NS5B polymerase inhibitor sofosbuvir. We first found that IFN enhances similarly ( 10 -fold $)$ the expression of inactive/non-phosphorylated PKR in infected and noninfected cells (Fig. 3A). In the absence of IFN, PKR levels in infected cells were slightly superior to those in non-infected cells ( 2-fold), suggesting that HCV replication in Huh7.5.1 cells up-regulates PKR expression (Fig. 3A). In contrast to $\mathrm{HCV}$-infected cells, we found that IFN fails to trigger PKR activation/phosphorylation in non-infected cells (Fig. 3A) ( 12-fold difference), suggesting that the presence of the virus is required for optimal PKR activation. As above, we found that all anti-HCV agents prevent PKR activation/ phosphory-lation in infected cells (5-6-fold decrease) and have no effect on the expression of inactive/non-phosphorylated PKR (Fig. 3A). The expression of non-phosphorylated and phosphorylated STAT1 is similarly up-regulated by IFN in infected and non-infected cells ( 13-fold increase) (Fig. 3A). Moreover, anti-HCV agents have no effect on the IFN-induced expression of non-phosphorylated and phosphorylated STAT1 (Fig. 3A). As expected alisporivir, daclastavir, sofosbuvir and IFN, by inhibiting JFH-1 replication, decrease NS5A expression (Fig. 3A). Together, these findings suggest that the IFN-mediated activation occurs only for specific components of the IFN response during an established infection.

\section{Suppression of HCV Replication Precludes PKR Activation}

Previous work suggested that the recognition of dsRNA by PKR induces its dimerization and activation by autophosphorylation [52]. Moreover, Targett-Adams et al. nicely showed that the replication of the $\mathrm{HCV}$ genome involves the generation of dsRNA replicative intermediates [53]. Based on these observations, we postulated that PKR activation/phosphorylation depends on the accumulation of HCV dsRNA during viral replication. In this scenario, blocking HCV replication by CypI or DAAs should suppress viral dsRNA accumulation and therefore PKR activation. To test this hypothesis, we took advantage of poly I:C, a structurally synthetic analog of dsRNA [54]. Poly I:C triggers PKR activation/phosphorylation in non-infected cells (Fig. 3B), suggesting that PKR recognizes the dsRNA mimic and that this recognition mediates its activation/ phosphorylation. The degree of PKR activation by poly I:C was less profound than that by $\mathrm{HCV}$, but was nevertheless significant (Fig. 3B). In contrast to the HCV-mediated PKR activation/phosphorylation, alisporivir did not suppress the poly I:C-mediated PKR activation/phosphorylation (Fig. 3B). This is in agreement with our hypothesis of work that inhibiting HCV replication prevents viral dsRNA accumulation and therefore precludes PKR activation/phosphorylation.

\section{DISCUSSION}

In this study, we investigated the possibility that CypI specifically modulate the IFN response to $\mathrm{HCV}$. We first found that the CypI alisporivir prevents PKR activation/phosphorylation in IFN $\alpha$-treated $\mathrm{HCV}$-infected cells. The inhibitory effect was also observed using type II and III IFNs as well as subgenomic replicon cells. We found that several CypI and various DAAs including protease, polymerase and NS5A inhibitors all suppress the IFNmediated PKR activation/phosphorylation in $\mathrm{HCV}$-infected cells. Moreover, we showed that IFN does not trigger PKR activation/phosphorylation in cells lacking $\mathrm{HCV}$. We also found that the dsRNA mimic poly I:C can substitute HCV for PKR activation/phosphorylation and that anti-HCV agents fail to prevent this activation. Based on these findings, we propose the following model (Fig. 4). IFN enhances the expression of the inactive/non-phosphorylated form of PKR. Newly expressed inactive PKR molecules recognize and bind to HCV dsRNA intermediates, which accumulate in the cytoplasm during viral replication when cells are cultured in the absence of anti-HCV agents. The binding of PKR to HCV dsRNA at the IRES [46] mediates PKR dimerization and autophosphorylation. In the presence of anti-HCV agents, viral replication is stopped and HCV dsRNA accumulation suppressed. In the absence of HCV dsRNA, no PKR activation/phosphorylation can occur.

Our model is in accordance with previous work of Thomis and Samuel who demonstrated using a mixture of wild-type and kinase-deficient PKR (K296R) proteins that PKR can be autophosphorylated in an intermolecular manner [55]. This model is also in accordance with recent studies from the Chisari and the Meurs labs, which elegantly demonstrated that early $\mathrm{HCV}$ infection not only triggers PKR induction and activation, but also attenuates the induction of ISG protein expression despite normal induction of ISG mRNAs [56, 57]. Moreover, they showed that when PKR expression is down-regulated by shRNA in IFN-treated infected cells, ISG protein induction is restored to normal levels and IFN antiviral effect is enhanced. Our findings, which are in accordance with those observations, further suggest that HCV exploits PKR activation to counteract the IFN response by preferentially suppressing the translation of ISGs, at least in the JFH-1 cell culture infection system. 


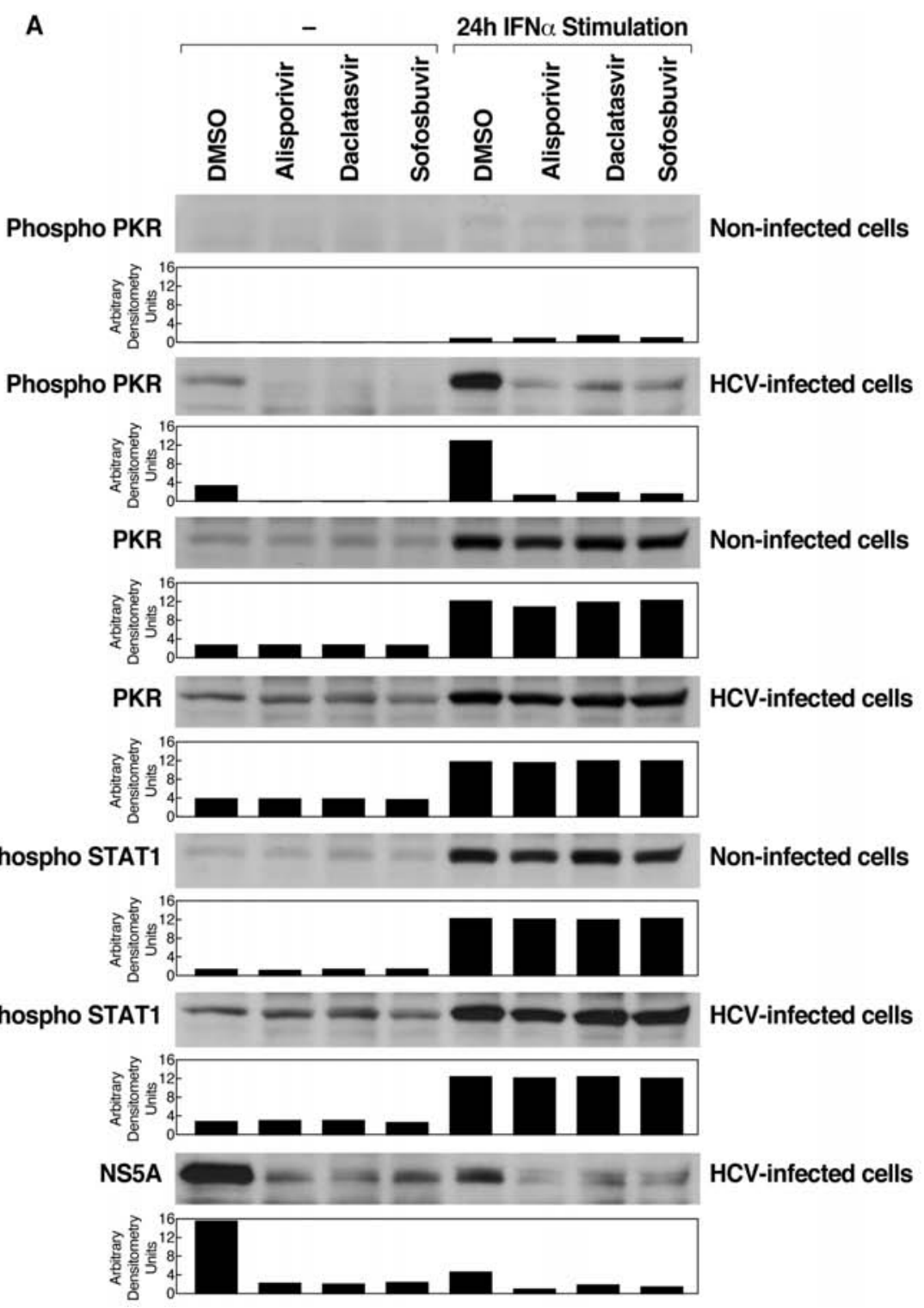

B
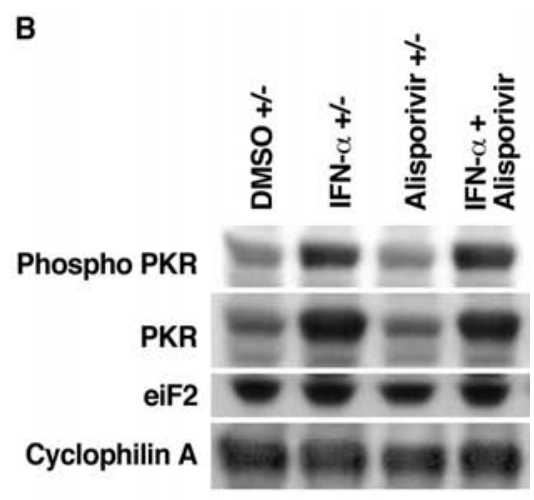

Cyclophilin A . 


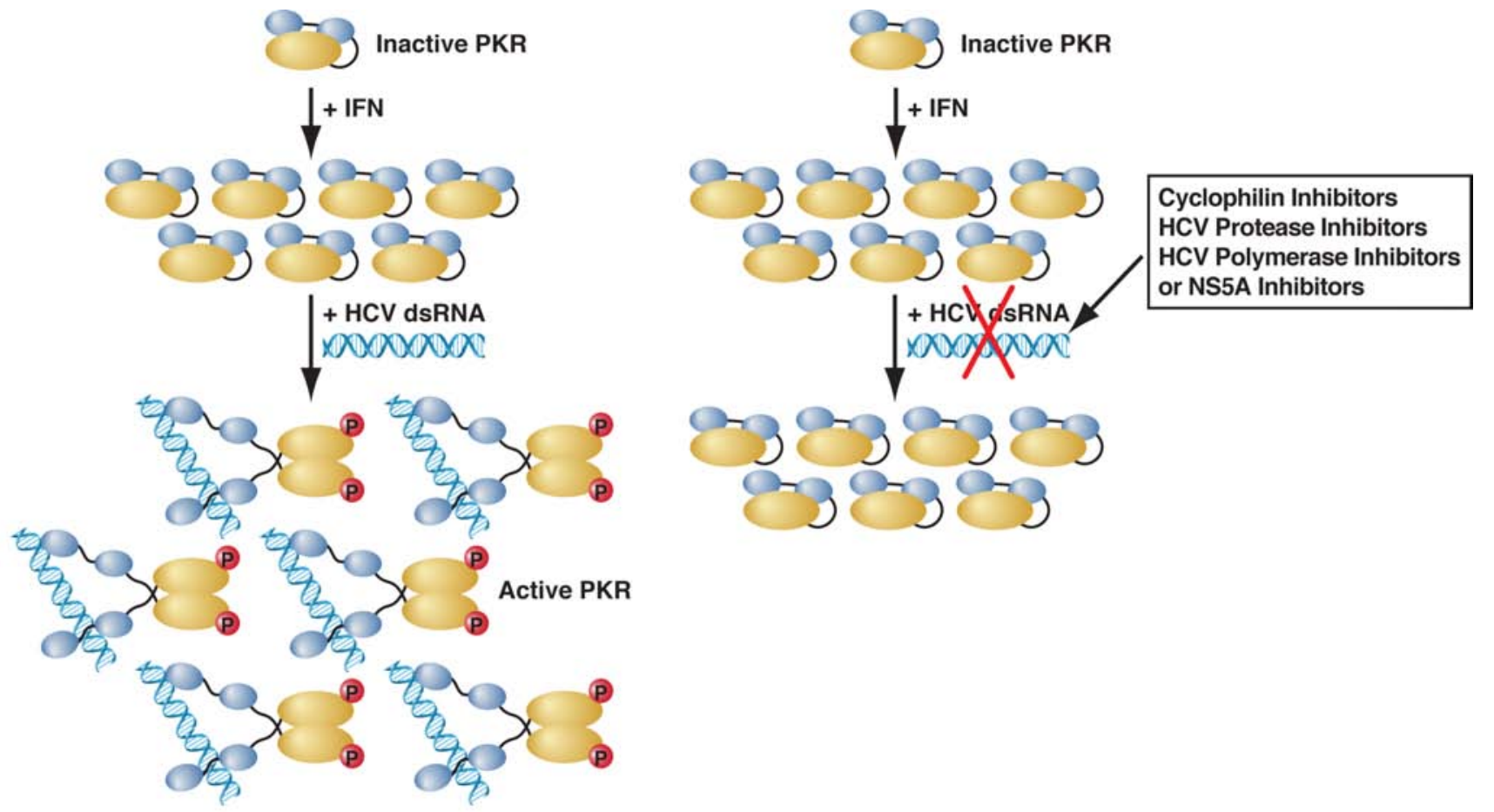

Fig. (4). Model for the effect of CypI and DAAs on the HCV-dependent activation of IFN-induced PKR. (Left) HCV replication in hepatoma cells leads to an accumulation of dsRNA intermediates. During this established infection, PKR is slightly expressed and activated. In response to IFN, infected cells overexpress the inactive form of PKR, which is subsequently activated/phosphorylated upon the recognition of and binding to viral dsRNA. (Right) The addition of anti-HCV agents either CypI or DAAs stops viral dsRNA accumulation resulting in the prevention of the activation of IFN-induced PKR.

the data presented here were generated using Huh7.5.1 cells. However, we conducted similar experiments with Huh7.5 and Huh7 cells and obtained comparable results (data not shown to avoid redundancy). The main reason why we present only data obtained using Huh7.5.1 cells created by the Chisari lab (49) is because this cell line is uniquely design to recapitulate all $\mathrm{HCV}$ infection and replication events that occur in hepatocytes. JFH-1-transfected Huh7.5.1 cells release up to $10^{5}$ focus-forming units per $\mathrm{mL}$. This number is 50-fold higher as compared to Huh7 cells. The difference appears to be due in part to higher permissiveness of Huh7.5.1 cells. Several features may explain this high permissiveness: i) Huh7.5.1 cells have a defect in the RIG-I pathway, making them less responsive to intracellular viral dsRNA; ii) they may express more numerous or "adequate" cell surface receptors mediating more efficacious $\mathrm{HCV}$ attachment and entry; or iii) virus assembly and budding is more effective in these cells.

Our study, which demonstrates that IFN fails to trigger PKR activation in non-infected cells and that both CypI and DAAs block PKR activation in HCV-infected cells, strongly supports the concept that suppression of viral replication results in the prohibition of the activation of IFN-mediated PKR during an established HCV infection. Our data also suggest that CypI in this experimental in vitro $\mathrm{HCV}$ infectivity design do not exert an action distinct from that of DAAs, at least in terms of IFN-induced PKR activation.

In conclusion, our study indicates that CypI do not have a unique effect on PKR activation, but rather the suppression of $\mathrm{HCV}$ replication by any anti-HCV agents, abrogates PKR activation. It also suggests that HCV exploits its dsRNA intermediates to trigger PKR activation that leads to suppression of translation of potentially antiviral ISGs that would otherwise endanger its survival. This study may also suggest that HCV hijacks PKR from the cellular antiviral machinery to weaken both the IFN-mediated antiviral response and IFN induction itself. Nevertheless, further work is required to determine whether PKR activation by $\mathrm{HCV}$ plays both a proviral and antiviral role during the IFN response depending on the phase of the infection (i.e., early versus established infection). It is important to re-emphasize that in this experimental infection cell culture system, IFN inhibits HCV replication despite PKR activation. We thus do not know at this stage whether the IFN-induced and HCVdependent PKR activation is beneficial or detrimental to the virus. It would be extremely interesting to examine the degree of hepatic PKR expression and activation in HCVinfected patients prior to, during and after either an IFN treatment or an IFN-free regimen composed of a combination of DAAs and CypI.
ABBREVIATIONS
CypA = Cyclophilin $\mathrm{A}$
CypI = Cyclophilin inhibitors
DAA = Direct-acting antivirals
dsRNA $=$ Double-stranded RNA
IFN $\alpha=$ Interferon $\alpha$ 


$$
\begin{aligned}
& \text { RBV = Ribavirin } \\
& \text { GT = Genotype } \\
& \text { HTA = Host-targeting antivirals } \\
& \text { ISGs }=\text { IFN-stimulated genes } \\
& \text { eIF22 = Eukaryotic initiation factor } 2 \\
& \mathrm{PKR}=\text { Protein kinase } \mathrm{R} \\
& \text { ISDR = IFN sensitivity-determining region } \\
& \text { IRES = Internal ribosome entry site } \\
& \text { poly I:C = Polyinosinic:polycytidylic acid } \\
& \text { NPT II = Neomycin phosphotransferase II } \\
& \text { IRF }=\text { IFN regulatory factor } \\
& \mathrm{NF}-\kappa \mathrm{B}=\text { Nuclear factor kappa-light-chain-enhancer of } \\
& \text { activated B cells } \\
& \text { OAS1 = Oligoadenylate synthetase } 1 \\
& \text { STAT = Signal Transducer And Activator Of } \\
& \text { Transcription }
\end{aligned}
$$

\section{CONFLICT OF INTEREST}

The authors confirm that this article content has no conflict of interest.

\section{ACKNOWLEDGEMENTS}

We thank Drs. Wakita and Chisari for Huh-7.5.1 and GT2a subgenomic JFH-1 replicon cells, Dr. Rice for the 9E10 anti-NS5A antibody, Drs. Bukh and Rice for the GT3a and 4a subgenomic JFH-1 replicon cells, Drs. Wakita and Pietschmann for the genomic Luc-JFH-1 plasmid, Novartis for alisporivir and Drs. Wilkinson and Gregory for cyclosporine A, sanglifehrins A and B. This work was supported by the U.S. Public Health Service grant no. AI087746 from the National Institute of Allergy and Infectious Diseases (NIAID). This is publication no. 25094 from the Department of Immunology \& Microbial Science, The Scripps Research Institute, La Jolla, CA.

\section{REFERENCES}

[1] Dienstag JL, McHutchison JG. American Gastroenterological Association technical review on the management of hepatitis $\mathrm{C}$. Gastroenterology 2006; 130: 231-64; quiz 214-237.

[2] Alter MJ. Epidemiology of hepatitis $\mathrm{C}$ virus infection. World $\mathrm{J}$ Gastroenterol 2007; 13: 2436-41.

[3] Soriano V, Madejon A, Vispo E, et al. Emerging drugs for hepatitis C. Expert Opin Emerg Drugs 2008; 13: 1-19.

[4] Shepard CW, Finelli L, Alter MJ. Global epidemiology of hepatitis C virus infection. Lancet Infect Dis 2005; 5: 558-67.

[5] Armstrong GL, Wasley A, Simard EP, McQuillan GM, Kuhnert WL, Alter MJ. The prevalence of hepatitis $\mathrm{C}$ virus infection in the United States, 1999 through 2002. Ann Intern Med 2006; 144: 70514.

[6] Cross TJ, Antoniades CG, Harrison PM. Current and future management of chronic hepatitis C infection. Postgrad Med J 2008; 84: $172-6$

[7] Manns MP, Wedemeyer H, Cornberg M. Treating viral hepatitis C: efficacy, side effects, and complications. Gut 2006; 55: 1350-59.

[8] Simmonds P, Bukh J, Combet C, et al. Consensus proposals for a unified system of nomenclature of hepatitis $\mathrm{C}$ virus genotypes. Hepatology 2005; 42: 962-73.

[9] Sy T, Jamal MM. Epidemiology of hepatitis C virus (HCV) infection. Int J Med Sci 2006; 3: 41-6.

[10] Tong MJ, Reddy KR, Lee WM, et al. Treatment of chronic hepatitis $\mathrm{C}$ with consensus interferon: a multicenter, randomized, controlled trial. Consensus Interferon Study Group. Hepatology 1997; 26: 747-54.

[11] Fischer G, Gallay P, Hopkins S. Cyclophilin inhibitors for the treatment of HCV infection. Curr Opin Investig Drugs 2010; 11: 911-8.

[12] Gallay PA. Cyclophilin inhibitors: a novel class of promising hosttargeting anti-HCV agents. Immunol Res 2012; 52: 200-10.

[13] Lin K. Development of novel antiviral therapies for hepatitis C virus. Virol Sin 2010; 25: 246-66.

[14] Pawlotsky JM. New antiviral agents for hepatitis C. F1000 Biol Rep 2012; 4: 5.

[15] Pockros PJ. New direct-acting antivirals in the development for hepatitis C virus infection. Ther Adv Gastroenterol 2010; 3: 191202.

[16] Vermehren J, Sarrazin C. New hepatitis C therapies in clinical development. Eur J Med Res 2011; 16: 303-14.

[17] von Hahn T, Ciesek S, Manns MP. Arrest all accessories-inhibition of hepatitis $\mathrm{C}$ virus by compounds that target host factors. Discov Med 2011; 12: 237-44

[18] Lin K, Gallay P. Curing a viral infection by targeting the host: the example of cyclophilin inhibitors. Antiviral Res 2013; 99: 68-77.

[19] Gallay PA, Lin K. Profile of alisporivir and its potential in the treatment of hepatitis C. Drug Des Dev Ther 2013; 7: 105-15.

[20] Flisiak R, Horban A, Gallay P, et al. The cyclophilin inhibitor Debio-025 shows potent anti-hepatitis $\mathrm{C}$ effect in patients coinfected with hepatitis $\mathrm{C}$ and human immunodeficiency virus. Hepatology 2008; 47: 817-26.

[21] Hopkins S, Dimassimo B, Rusnak P, et al. The cyclophilin inhibitor SCY-635 suppresses viral replication and induces endogenous interferons in patients with chronic HCV genotype 1 infection. J Hepatol 2012; 57: 47-54.

[22] Lawitz E, Godofsky E, Rouzier R, et al. Safety, pharmacokinetics, and antiviral activity of the cyclophilin inhibitor NIM811 alone or in combination with pegylated interferon in $\mathrm{HCV}$-infected patients receiving 14 days of therapy. Antiviral Res 2011; 89: 238-45.

[23] Herrmann E, Kafer A, Flisiak R, Nicolas-Metral V, Zeuzem S, Crabbe R. PK-PD modeling of viral kinetics during treatment with Debio-025 plus pegylated interferon $\alpha-2 \mathrm{a}$ in treatment-naive HCV patients. J Hepatol 2009; 50(S1): 344

[24] Flisiak R, Feinman SV, Jablkowski M. et al. The cyclophilin inhibitor debio 025 combined with Peg-IFN2a significantly reduces viral load in treatment naïve hepatitis C patients. Hepatology 2009; 49: $1460-8$.

[25] Flisiak R, Pawlotsky JM, Crabbe R, et al. Once daily alisporivir (DEB025) plus peg-IFN-Alfa-2A/Ribavirin results in superior sustained virologic response (SVR24) in chronic hepatitis C genotype 1 treatment-naïve patients-The ESSENTIAL study. J Hepatol 2011; 55: Abstract 190.

[26] Pawlotsky JM, Sarin SK, Foster GR, et al. Alisporivir plus ribavirin is highly effective as interferon-free or interferon-add-on regimen in previously untreated HCV-G2 or G3 patients: SVR12 results from VITAL-1 phase 2b study. J Hepatol 2012; 56: Abstract 1405.

[27] Pawlotsky JM, Sarin SK, Foster GR, et al. Alisporivir plus ribavirin achieves high rates of sustained HCV clearance (SVR24) as interferon (IFN)-free or IFN-add-on regimen in treatment-naive patients with HCV GT2 or GT3: Final results from VITAL-1 study. Hepatology 2012; Suppl.56 (4),309A (Abstract 233).

[28] Alberti M, Chuang WL, Flisiak R et al. Alisporivir (ALV) plus peg-interferon/ribavirin (PR) in HCV G1 treatment-experienced patients achieves primary endpoint with superior efficacy at treatment week 12 compared to retreatment with PR. J Hepatol 2012; 56: Abstract 1406.

[29] Davis G, Kao J, Alberti A, et al. Alisporivir (ALV) plus Peginterferon/Ribavirin (P/R) Achieves High On-Treatment Undetectable HCV RNA Levels Among the Most Difficult to Treat HCV G1 Patients. Results of a Planned Treatment Week 24 Interim Analysis of a Randomized, Double Blind, Placebo Controlled Trial (FUNDAMENTAL study). 63rd Annual Meeting of the American Association for the Study of Liver Diseases, 2012, Nov 9-12; Boston MA 2012.

[30] Griffel L, Bao W, Orsenigo R,et al. Interferon (IFN)-free alisporivir has a better overall safety profile compared to IFNcontaining treatment: a pooled analysis of the ALV development program. J Hepatol 2013; 58, (Suppl 1): 336-7. 
[31] Liu Z, Yang F, Robotham JM, Tang H. Critical role of cyclophilin $\mathrm{A}$ and its prolyl-peptidyl isomerase activity in the structure and function of the hepatitis C virus replication complex. J Virol 2009; 83: 6554-65.

[32] Kaul A, Stauffer S, Berger C, et al. Essential role of cyclophilin A for hepatitis $\mathrm{C}$ virus replication and virus production and possible link to polyprotein cleavage kinetics. PLoS Pathog 2009; 5(8): e1000546.

[33] Chatterji U, Bobardt MD, Selvarajah S, et al. The isomerase active site of cyclophilin A is critical for hepatitis $\mathrm{C}$ virus replication. $\mathrm{J}$ Biol Chem 2009; 284: 16998-17005.

[34] Dorner M, Horwitz JA, Donovan BM, et al. Completion of the entire hepatitis $\mathrm{C}$ virus life cycle in genetically humanized mice. Nature 2013; 501: 237-41.

[35] Harding MW, Handschumacher RE. Cyclophilin, a primary molecular target for cyclosporine. Structural and functional implications. Transplantation 1988; 46(Suppl 2): 29-35.

[36] Hanoulle X, Badillo A, Wieruszeski JM, et al. Hepatitis C virus NS5A protein is a substrate for the peptidyl-prolyl cis/trans isomerase activity of cyclophilins A and B. J Biol Chem 2009; 284: 13589-601.

[37] Fernandes F, Ansari IU, Striker R. Cyclosporine inhibits: A direct interaction between cyclophilins and hepatitis C NS5A. PLoS One 2010; 5: e9815.

[38] Chatterji U, Lim P, Bobardt MD, et al. HCV resistance to cyclosporin A does not correlate with a resistance of the NS5Acyclophilin A interaction to cyclophilin inhibitors. J Hepatol 2010; 53: 50-6.

[39] Coelmont L, Hanoulle X, Chatterji U, et al. DEB025 (Alisporivir) inhibits hepatitis $\mathrm{C}$ virus replication by preventing a cyclophilin $\mathrm{A}$ induced cis-trans isomerisation in domain II of NS5A. PLoS One 2010; 5: e13687.

[40] Verdegem D, Badillo A, Wieruszeski JM, et al. Domain 3 of NS5A protein from the hepatitis $\mathrm{C}$ virus has intrinsic alpha-helical propensity and is a substrate of cyclophilin A. J Biol Chem 2011; 286(23): 20441-54.

[41] Rosnoblet C, Fritzinger B, Legrand D, et al. Hepatitis C virus NS5B and host cyclophilin A share a common binding site on NS5A. J Biol Chem 2012; 287(53): 44249-60

[42] Samuel CE. Antiviral actions of interferon. Interferon-regulated cellular proteins and their surprisingly selective antiviral activities. Virology 1991; 183: 1-11.

[43] Gale M Jr, Blakely CM, Kwieciszewski B, et al. Control of PKR protein kinase by hepatitis $\mathrm{C}$ virus nonstructural 5A protein: molecular mechanisms of kinase regulation. Mol Cell Biol 1998; 18(9): 5208-18

[44] Huang L, Hwang J, Sharma SD, et al. Hepatitis C virus nonstructural protein 5A (NS5A) is an RNA-binding protein. J Biol Chem 2005; 280: 36417-28.
[45] Hwang J, Huang L, Cordek DG, et al. Hepatitis C virus nonstructural protein $5 \mathrm{~A}$ : biochemical characterization of a novel structural class of RNA-binding proteins. J Virol 2010; 84: 1248091.

[46] Toroney R, Nallagatla SR, Boyer JA, Cameron CE, Bevilacqua PC. Regulation of PKR by HCV IRES RNA: importance of domain II and NS5A. J Mol Biol 2010; 400: 393-412.

[47] Wakita T, Pietschmann T, Kato T, et al. Production of infectious hepatitis $\mathrm{C}$ virus in tissue culture from a cloned viral genome. Nat Med 2005; 11: 791-6.

[48] Koutsoudakis G, Kaul A, Steinmann E, et al. Characterization of the early steps of hepatitis $\mathrm{C}$ virus infection by using luciferase reporter viruses. J Virol 2006; 80: 5308-20.

[49] Vrolijk JM, Kaul A, Hansen BE, et al. A replicon-based bioassay for the measurement of interferons in patients with chronic hepatitis C. J Virol Methods 2003; 110: 201-9.

[50] Zhong J, Gastaminza P, Cheng G, et al. Robust hepatitis C virus infection in vitro. Proc Natl Acad Sci USA 2005; 102: 9294-9.

[51] Lindenbach BD, Evans MJ, Syder AJ, et al. Complete replication of hepatitis C virus in cell culture. Science 2005; 309: 623-6.

[52] Gale M Jr, Katze MG. Molecular mechanisms of interferon resistance mediated by viral-directed inhibition of PKR, the interferon-induced protein kinase. Pharmacol Ther 1998; 78: 29-46.

[53] Targett-Adams P, Boulant S, McLauchlan J. Visualization of double-stranded RNA in cells supporting hepatitis C virus RNA replication. J Virol 2008; 82: 2182-95.

[54] Lindh HF, Lindsay HL, Mayberry BR, Forbes M. Polyinosiniccytidylic acid complex (poly I:C) and viral infections in mice. Proc Soc Exp Biol Med 1969; 132: 83-7.

[55] Thomis DC, Samuel CE. Mechanism of interferon action: evidence for intermolecular autophosphorylation and autoactivation of the interferon-induced, RNA-dependent protein kinase PKR. J Virol 1993; 67: 7695-700.

[56] Garaigorta U, Chisari FV. Hepatitis C virus blocks interferon effector function by inducing protein kinase $\mathrm{R}$ phosphorylation. Cell Host Microbe 2009; 6: 513-22.

[57] Arnaud N, Dabo S, Maillard P, et al. Hepatitis C virus controls interferon production through PKR activation. PLoS One 2010; 5(5): e10575.

[58] Watashi K, Daito T, Sluder A, Borroto-Esoda K, Wakita T. Cyclophilin inhibitors potentiate interferon signaling through diminished PKR phosphorylation in $\mathrm{HCV}$-infected cells. 48th Annual Meeting of the European Association for the Study of the Liver 2013; 58, S632.

[59] Watashi K, Daito T, Sluder A, Nakajima S, Borroto-Esoda K, Wakita T. International Symposium on Hepatitis $\mathrm{C}$ Virus and Related Viruses, Melbourne. 2013; Novel regulation mechanism of interferon signaling by cyclophilin through modulation of PKR in HCV-infected cells. Abstract 32218.

(C) Bobardt et al.; Licensee Bentham Open.

This is an open access article licensed under the terms of the Creative Commons Attribution Non-Commercial License (http://creativecommons.org/licenses/ by-nc/3.0/) which permits unrestricted, non-commercial use, distribution and reproduction in any medium, provided the work is properly cited. 